\title{
The Nexus of Art and Science: Creating Art Medals in the Chemistry Lab, Using Graphite Molds
}

\author{
Megan Karjo \\ Constantina Sinani \\ Coryn Le \\ Mark A. Benvenuto \\ Department of Chemistry \& Biochemistry, \\ University of Detroit Mercy, Detroit, MI, USA
}

\begin{abstract}
The idea of graphing what is called the lead-tin eutectic point when alloys are produced from elemental lead and tin metals may be very common and established in chemistry and materials science laboratories. Producing art medals from the alloys that are made in such experiments is decidedly not. We will present how the lead-tin eutectic experiment can be performed in the chemistry lab, and how graphite blocks can be used as both mold and heat sink for the creation of art medals, utilizing metal alloys produced by students in the laboratory. The process brings together art, science and engineering in a single experience. We conclude that this has been found to be beneficial for students, yet is a technique that can be utilized by virtually any person who wishes to experiment and create with low-melting metal alloys.
\end{abstract}

Keywords: Alloy, Low-melting alloys, Eutectic, Art medal, Heat transfer

\section{Introduction}

The modern general chemistry laboratory usually offers a variety of phenomena to students, often those which have been studied extensively, and for which the results are well known. This tends to make it easy for students to perform an experiment, and for faculty members to grade it. Over the course of decades however, as these "canned" labs have evolved, it appears that experiments in which reduced metals are used and studied have faded from the curriculum. While there appears to be a number of low melting alloys that can be utilized as is or as purchased, or adjusted in composition, all of which are safe to work with, it appears nevertheless that 
laboratory experiments which use metals have been deleted from many chemistry curricula.

What is known as the lead-tin eutectic lab is a series of experiments in which a known amount of one metal, lead or tin, is melted, and some known mass of the other is added to the molten sample. The resulting alloy is quenched in cold water, dried, re-melted, and the temperature taken at the initial point of melting. A further portion of the second metal is added to the first alloy, and the quench and re-melt are repeated. In this manner, a student can gather enough data points that what is called the eutectic curve can be graphed with good accuracy. The melting points of elemental tin and elemental lead can be used as the terminal points on such a graph, with the $\mathrm{x}$-axis being the weight percent of one metal from $0-100 \%$ (which is $100-0 \%$ for the other metal), and the y-axis will be temperature. This experiment is often very instructive for students, because they approach it with the preconceived idea that the graph will be a straight line between the melting point of lead $\left(328^{\circ} \mathrm{C}\right)$ and the melting point of tin $\left(232^{\circ} \mathrm{C}\right)$, which emphatically it is not. When metals of good quality and high purity are used, the eutectic point, which is approximately $55 \%$ tin, is $176^{\circ} \mathrm{C}$. When lower purity metals are used, such as lead or tin purchased from scrap yards, the eutectic point can be as low as $86^{\circ} \mathrm{C}$, as seen in the past by the main author.

One of the end results of performing this experiment in a chemistry lab is leftover metal of no specific alloy. The idea of using such products of any experiment that produces this, or any low-melting metal alloys, as the start point for the production of art medals appears to be without literature precedent. At least, after a thorough search, we have found no references to this with the exception of our own, recent work.[1-5].

\section{The technique of casting medals}

Graphite has been used for generations to aid glassblowers in removing heat from semi-molten pieces of glass while they are being blown or shaped. This is because the heat capacity of graphite is high compared to many other solids. Near where the authors live is the henry Ford Greenfield Village - a museum that showcases arts and sciences of the past. Glass is still blown in a traditional manner there; and glassblowers roll semi-molten glass on graphite plates to cool the glass. The graphite absorbs the heat very well.[6]

In a general chemistry lab, the phenomenon we are trying to examine is one of a chemical change, or perhaps one involving heat transfer. The use of a graphite mold to cool molten metal is definitely a matter of heat transfer. Engineering students find this to be of particular interest, because tin-lead objects cast in a mold made entirely of graphite can be removed from a 3" cube of graphite in less than a minute and be cool to the touch.

Any number of objects can be made using a graphite mold, for example, parts for some larger, more complex machine. But such parts can be produced using other materials 
for the mold, and the heat transfer managed by some coolant applied to the mold. In the case of a graphite block, it can be carved directly, using any wood-carving or metal-carving tools, and used as both heat sink for the metal being poured, and mold for some design - in our case, for an art medal. We have pursued this in the freshmenlevel chemistry lab, allowing students to first create some design, often simply on a page in their lab notebook, then allowing them the time to carve into the graphite block. This may take more than one lab period, but student results are often quite good, especially considering that this is the first time virtually every student making a medal has done so.

This experiment can be considered an amazingly simple one, at least in terms of its chemistry. It is the production of a series of mixtures, as alloys are generally mixtures of two or more metal elements.[7] Since there is no further use for the metal alloys when the initial lead-tin eutectic experiment is complete, this metal becomes an ideal material to use for the creation of medals.

Graphite has a specific heat of $0.720 \mathrm{~J} / \mathrm{g}^{\circ} \mathrm{C}$. This is almost 1.6 times greater than that of iron, and almost 1.9 times greater than that of copper. These two elemental metals are used as points of comparison in terms of specific heat, because molds into which metal objects are poured are often made of such metals. Thus, molten metal must cool in such molds. Using graphite as a material for the mold, and using lead-tin alloys as the metal, means that heat can be transferred to the mold, resulting in objects medals - that can be removed from the molds in minutes, as opposed to hours or days.

The idea of using graphite as some form of heat sink certainly has an established history to it. As mentioned, glass blowers at the Henry Ford Greenfield Village in Dearborn, Michigan, USA, still use graphite plates to cool masses of semi-molten glass when they are shaping and creating objects. This is a part of the Greenfield Village experience that is seen by numerous visitors to the museum. [6] But there exists more than simply a niche use of graphite in a historical context. Graphite continues to be used in a variety of industrial applications. In this study, graphite blocks were purchased locally from a business, Graphite Products Corporation, which supplies to customers throughout the Midwest of the United States.[8]

The term "low melting alloy" is a somewhat flexible one, but from the point of view of the artist who sculpts and works with metals in creating objects, the term may be conveniently described as a metal that melts at a low enough temperature that it can be worked without having to employ the services of a foundry.[9] When working in brass or bronze, two traditional metal alloys for making art objects, some model of the object must be sent to a foundry, made into a mold using a variety of materials such as clays or resins, then poured. This can be time consuming, and may result in a finished product with which the artist finds some fault. When working with a graphite mold, which is very easy to carve, and an alloy that can be melted with a hot plate or a Bunsen burner flame, the finished object can be examined quickly, and corrections can also be made quickly if the artist so chooses. This process of pouring a medal, 
examining the finished product, and making changes as needed, can be repeated numerous times if the artist so chooses.

Thus, we believe we have developed a technique that is easy and direct for artists and art students to employ, that is an excellent experiment in the chemistry of solids, and that shows directly how heat is transferred, which is of interest to engineers and engineering students.

\section{Experimental steps}

The experimental procedure for the lead-tin eutectic lab, and creating alloys of the two metals, can be listed as follows:

Needed:

Lead metal

Tin metal

Thermometer or thermocouple

Crucible

Heat source - hot plate or Bunsen burner

Beaker of cold water

It is easiest to start by melting a pre-weighed piece or pieces of lead metal. The temperature does not need to be recorded in the class, because it is well-known and can be found on-line.

After the lead is molten, add a sample of tin metal of known mass.

Allow the alloy to melt.

Pour the molten alloy into cold water to solidify it.

Dry the pieces of alloy that have formed in the cold water.

Place the cooled alloy in the crucible, along with the thermometer bulb or thermocouple tip, ensuring the thermometer end does not touch the bottom of the crucible, and that it is in good contact with the solid pieces of alloy.

Heat the alloy until it is again molten. During this step, watch carefully and take the temperature reading at the first sign of any molten metal. Unlike water or other covalently bonded matter, there is no plateau of melting for these alloys. Thus, the temperature at which the first drop of metal appears will be lower than that at which the last bit of solid disappears. It is advantageous to have students work in pairs, so that one can spot the first drop of molten metal and the other can take the temperature.

After the alloy is entirely melted, add a further known mass of tin. 
Repeat steps 5 - 9 as many times as desired.

Following these steps it is not difficult to obtain five data points for different alloys. When adding the known values for the melting points of lead and tin, a graph can be made with the two known values serving as the $0 \%$ point and $100 \%$ point for each metal, as the farthest points of the graph.

Importantly, adding amounts of tin to lead in a successive manner produces graphs with a wide spread of percentages. We have found that if students pre-weigh five amounts of lead and five amounts of tin, the five data points obtained tend to be clustered towards the center of any graph that is produced. It seems to be common to use roughly the same size samples if indeed five sets of lead metal and tin metal are set aside before any alloys are made.

As well, experience has taught us that it is best to run this experiment in pairs, since it can be difficult to spot the moment at which an alloy first melts and at the same time correctly read a thermometer or thermocouple. The temperature rises quickly, especially with smaller samples $(10-20 \mathrm{~g})$, and students can miss the temperature at the point of first melting. It should be remembered that this is the first such experiment for many students, and thus they do not have extensive experience taking temperature readings while a material changes phase. Failure to take these readings properly tends to result in graphs that do not show the eutectic dip.

\section{Carving graphite blocks}

This aspect of the experiment does not have to be performed in the lab at the same time as the production of the lead-tin alloys. It can be performed over the remainder of a semester, since the metals that result from the eutectic experiment can easily be kept in the lab for later use. Our experience has taught us that many students prefer to work on their design over the course of several weeks, then pour a medal towards the end of the semester. Carving tools are provided in the class, but some students request to take their block back to their home because they or a relative has an extensive tool bench, which may include better carving tools than those available in the lab.

Because students generally have never carved in graphite before, we encourage them to wear older clothing, since graphite particles tend to blacken clothes and skin when it is being worked with. It washes off easily, but does stick to clothes until they can be washed. In the past, some students have chosen to wet their graphite block while carving it, to minimize the amount of graphite dust in their work area. This does work, but doing so means that metal should not be poured into the mold until it is completely dry. Pouring while it is wet tends to cause some bubbling on the surface of the finished medal. 


\section{Conclusions and future directions}

Lead-tin alloys have provided excellent results when working with low-melting alloys, and can be performed even in the first year or freshmen-level chemistry laboratory. We encourage students to work in pairs when taking readings of melting points to maximize the accuracy of their data, and thus gather data that will well illustrate the lead-tin eutectic graph.

We have found that the resultant metal is an excellent source of material from which to create art medals. They are easy to produce, easy to work with, and can be poured in graphite molds even by students who have had no prior experience.

We intend to continue our exploration of low-melting alloys using others, such as Wood's metal fusible alloy, or Onion's alloy,[10,11] which we hope will provide results that are equally intriguing and educational. We also believe that the composition of such low-melting alloys can be adjusted for results that are more in line with what the artist and art student desire, specifically, adding more tin to such alloys.[12]

We have found that this experiment draws the interest of students, serves as an excellent example of solid-state chemistry that can be performed as early as the freshman year of college or university, and has strong connections between the sciences and engineering, and the arts. We encourage others to adopt this in their own teaching laboratories.

\section{References}

[1] Benvenuto, M.A., Maurice, S., \& Paulsen, B. (2016). The Nexus of Art and Science: Cast medals graphite molds. Médailles, 153-156.

[2] Pothoof, J., Baker, C. \& Benvenuto, M.A. (2018). The Nexus of Art and Science II: Making Two-Sided Medals with Graphite Molds. Médailles, 247-249.

[3] Tieu Ngo, T., Rogers, A., Arce, W., Baker, C. \& Benvenuto, M.A. (2019). Metals chemistry for the classroom, K-16: A tutorial on low-melting alloys. In Proceedings of the 257th National Meeting of the American Chemical Society, (HIST8). Washington, DC.

[4] Seagram, S., Hedglin, S., Gallant, A., Aufdemberge, W., Tokarz, N., Plieth, S., Blackwell, C., Tieu Ngo, T., Rogers, A., Kosmas, S.P., \& Benvenuto, M.A. (2019) Creating low-melting metal alloys: Connections between chemistry and arts classes. In Proceedings of the Central Regional Meeting of the American Chemical Society 2019, (CERM51), Midland, MI.

[5] Kosmas, S. \& Benvenuto, M.A. (2020). Making metals and making medals: A nexus of science and art. In Proceedings of the 67th annual conference of the Michigan Science Teachers Association, Lansing, MI.

[6] Henry Ford Greenfield Village, (2020). [Online] Available: thehenryford.org/current-events/calendar/glass-blowing-demonstration.

(10 July 2020) 
[7] Greenwood, N.N. and Earnshaw, A. (1997). Chemistry of the Elements. (2 ${ }^{\text {nd }}$ ed.). Amsterdam: Butterworth-Heinemann, Elsevier.

[8] Graphite Products Corporation, (2020). [Online] Available: graphiteproductscorp.com. (10 July 2020)

[9] Belmont Metals, (2020). [Online] Available: "Low melting-point (fusible) alloys," downloadable as pdf. (10 July 2020)

[10] (No author listed). (1888). Biography, Barnabas Wood. Transactions of the Medical Society of the County of Albany, 379-380.

[11] Jensen, W.B. (2010). The Origin of the Name 'Onion's Fusible Alloy'. Journal of Chemical Education, 87, 1050-1051.

[12] (No author listed) 'Caring for your Lead, Coins \& Medals.' (2020). [Online] Available: Victoria and Albert Museum, vam.ac.uk/content/articles/c/caringfor-your-lead-coins-medals/ (25 June 2020) 